\title{
Extrapolation of Laminar Flame Speeds from Stretched Flames:
}

\section{Role of Finite Flame Thickness}

\author{
Wenkai Liang ${ }^{1}$, Fujia $W u^{1}$ and Chung K. Law ${ }^{1,2 *}$ \\ ${ }^{I}$ Department of Mechanical and Aerospace Engineering, Princeton University, \\ Princeton, NJ 08544, USA \\ ${ }^{2}$ Center for Combustion Energy, Tsinghua University, Beijing 100084, China
}

\begin{abstract}
:
Recognizing the persistent inaccuracy of existing linear and nonlinear expressions to extract laminar flame speeds from experimental data of stretched flame speeds, and the essential and independent role of the flame thickness, in addition to the laminar flame speed, in describing the coupled diffusive and reactive response of the laminar flame structure and propagation, we have adopted the finite flame thickness expression of Frankel and Sivashinsky in the extrapolation. Using numerically generated stretched flame data of the outwardly expanding spherical flame for both lean and rich hydrogen/air and $n$-heptane/air mixtures, it is demonstrated that the finite thickness expression yields not only improved extraction of the laminar flame speed, it also collaterally yields the laminar flame thickness as well as the Markstein length. Additionally, we have examined the cause for the inaccuracy of the pervious linear and nonlinear extrapolation expressions, the effects of pressure on the extrapolation results, and the robustness of the various expressions in accounting for the experimental errors.
\end{abstract}

Keywords: Expanding Spherical Flame, Laminar Flame Speed, Stretch Extrapolation, Flame Thickness

\section{Introduction}

Laminar flame speed, defined as the steady and adiabatic propagation speed of a one-dimensional, planar, flame in the doubly infinite domain [1], is a fundamental property of a combustible mixture. Consequently an accurate knowledge of its value not only is of practical utility in the assessment of the mixture's burning rate and flammability, it can also be used to develop/interrogate theories/models of the fundamental processes of diffusion and reaction constituting the flame structure. In particular, various experimental methods have been developed to measure its value in different flame 
configurations, such as the Bunsen flame, burner-stabilized flat flame, counterflow/stagnation flame and spherical expanding flame [2]. Since some of these flames are aerodynamically stretched, the associated stretch effects need to be subtracted out to yield the unstretched value. The subtraction usually involves extrapolating the stretched flame speeds at various stretch rates to vanishing stretch rate, with the extrapolation guided by expressions derived with various rigors through asymptotic analysis of flames subjected to a generalized stretch rate, $K$. The need to achieve ever greater accuracy in the extrapolation, so as to reduce the uncertainty in the extraction of the fundamental kinetics information, has recently led to the use of more detailed extrapolation expressions which would involve nonlinear as well as multiple expansion terms [3-5]. In spite of such efforts, several recent studies [6-9] have nevertheless noted that substantial uncertainties and errors in extrapolation still emerge for all the existing extrapolation expressions, particularly when the flame Lewis number, $L e$, is either too small or too large, as for very lean or rich hydrogen/air flames [6,7]. Since $L e$ is functionally coupled to the stretch rate, this inaccuracy in turn suggests that the extrapolation functions, even already quite detailed, are still inadequate to guide the extrapolation with sufficient accuracy. Consequently we are led to the inevitable need to revisit the adequacy of the key assumptions underlying these extrapolation expressions, which were invariably derived through asymptotic analyses, and to implement functionally more appropriate revisions if necessary.

Since flame propagation is controlled by the coupling of two fundamental processes of diffusion and chemical reaction, each with its own distinct rate, $\alpha$ and $w$, respectively, where $\alpha$ is a characteristic thermal diffusivity and $w$ a characteristic reaction rate, there should also be two independent response functions [1], which can be selected as the flame speed $S_{l}^{0}$ and the flame thickness $\delta^{0}$, given by the product and ratio of $\alpha$ and $w$, namely, 


$$
S_{l}^{0} \propto \sqrt{\alpha w}, \delta^{0} \propto \sqrt{\frac{\alpha}{w}}
$$

with the proportionality parameter being dependent on the mixture density. It is then recognized that so far only $S_{l}^{0}$ is treated as the extrapolation target in existing linear [10] or nonlinear [3-5] extrapolation expressions, with no explicit and independent extraction of $\delta^{0}$. This is because most previous extrapolation expressions were derived in the limit of reaction and flame sheets [11], for which the reaction zone is asymptotically thin compared to the preheat zone, which in turn is thin compared to the length scale of the hydrodynamic flow field, represented for example by the flame radius, $r_{f}$, for the spherical flame configuration. Consequently, the resulting $S_{l}^{0}$ describes the motion of the entire flame interface relative to the flow field, and the asymptotic results yield only $S_{l}^{0}$ (and the Markstein length to describe stretch sensitivity) as the "eigenvalue" to be determined, with $\delta^{0}$ being related to $S_{l}^{0}$ and a diffusivity instead of independently specified.

In addition to the functional deficiency of the flame-sheet analyses, it is also reasonable to expect that there are situations in which deviations from the flame-sheet limit can be substantial, due to high stretch rates and curvature, such that the effect of finite flame thickness could quantitatively affect the extrapolated results. Furthermore, computational simulation and experiments have amply demonstrated the complexity of the fundamental flame structure in that chemical reactivity including substantial heat release can intrude into the low-temperature, "preheat" zone of the flame through, for example, the back diffusion of the highly mobile $\mathrm{H}$ atom and the subsequent temperature-insensitive, exothermic, recombination reaction of $\mathrm{H}+\mathrm{O}_{2}+\mathrm{M} \rightarrow \mathrm{HO}_{2}+\mathrm{M}$.

Recognizing the need to treat the flame thickness $\delta^{0}$ as an independent fundamental property of the flame, even though it is not as precisely defined as the laminar flame speed $S_{l}^{0}$, we shall revise the 
extrapolation procedure by allowing the flame to have a finite $\delta^{0}$, which is to be consistently determined together with $S_{l}^{0}$. We shall adopt the expanding spherical flame as a prototypical configuration to conduct our investigation, as this configuration has been extensively studied recently in terms of the extrapolation issue, and since an explicit asymptotic expression allowing a finite $\delta^{0}$ has also been derived in the literature. In the following we shall first review and discuss the various extrapolation expressions and the relations between them. We shall next conduct detailed simulation of the outwardly expanding flame under different conditions, and then perform extrapolations using these simulated results as data, and show convincingly that the proposed extrapolation expression with finite flame thickness leads to the closest extraction of the laminar flame speeds. We shall also present the flame thicknesses and the Markstein lengths which are collaterally determined with the laminar flame speeds, hence providing reasonably adequate closure to the algorithm needed to extract the global fundamental laminar flame properties from experimentally measured stretched laminar flame speeds.

\section{Extrapolation Expressions}

In the extrapolation process, the flame front history $r_{f}(t)$ is first experimentally acquired using highspeed cine-photography, frequently Schlieren-based, indicating the position of maximum heat release or the maximum temperature gradient [1]. Using $r_{f}(t)$, the stretched flame speed with respect to the burned mixture and the stretch rate of the expanding spherical flame can be derived as $S_{b}=\left(d r_{f}\right) / d t$ and $K=$ $\left(2 / r_{f}\right) d r_{f} / d t$. Using $S_{b}$ and $K$ (or $\left.r_{f}\right)$, the unstretched laminar flame speed, $S_{b}^{0}$, and the Markstein length, $l_{b}$ with respect to the burned mixture, have been extrapolated. The simplest extrapolation, first suggested in [10] for the counterflow configuration, is the linear expression (LE), given by:

$$
\frac{S_{b}}{S_{b}^{0}}=1-\frac{l_{b} K}{S_{b}^{0}}
$$


The linear extrapolation, while convenient to apply, not only requires extensive range of data for fitting, but it also becomes increasingly inaccurate for small stretch rates. Subsequently Kelley and Law [3] used a nonlinear expression (NE)

$$
\left(\frac{s_{b}}{s_{b}^{0}}\right) \ln \left(\frac{s_{b}}{s_{b}^{0}}\right)=-\frac{2 l_{b}}{r_{f}}
$$

asymptotically derived by Ronney and Sivashinsky [12] based on quasi-steady and weak flame perturbation assumptions.

Equations (2) and (3) allow the extrapolation of two global flame parameters, namely $S_{b}^{0}$ and $l_{b}$, but not the characteristic flame thickness $\delta^{0}$, as discussed earlier. Revisiting previous theoretical works on expanding spherical flames, we note that Frankel and Sivashinsky derived the asymptotic solution for the adiabatic, outwardly propagating spherical flame, allowing for finite flame thickness [13], and later presented by Chen and $\mathrm{Ju}[14]$, as

$$
\left(U+\frac{2}{R}\right) \ln \left(U+\frac{2}{R}\right)=\frac{Z-2}{R}\left(\frac{1}{L e}-1\right)
$$

where $U, R, L e, Z$ are, respectively, the normalized flame speed, the normalized flame radius, the Lewis number, and the Zel'dovich number. This formula was mostly presented in theoretical analysis [13, 14] rather than used as an extrapolation expression. From the analysis in Ref. [1], the Markstein number, $M a$, which is the Markstein length, $l_{b}$, normalized by the adiabatic planar flame thickness, $\delta^{0}$, can be expressed as $M a=L e^{-1}-Z / 2\left(L e^{-1}-1\right)$. Hence, in dimensional form, Eq. (4) can be written as 


$$
\left(\frac{s_{b}}{s_{b}^{0}}+\frac{2 \delta^{0}}{r_{f}}\right) \ln \left(\frac{s_{b}}{s_{b}^{0}}+\frac{2 \delta^{0}}{r_{f}}\right)=-\frac{2\left(l_{b}-\delta^{0}\right)}{r_{f}}
$$

This formula is similar to the nonlinear expression of Eq. (3), except for the additional term corresponding to the finite thickness $\delta^{0}$, which vanishes in the limit of $\delta^{0} / r_{f} \rightarrow 0$. Since this expression allows for the finite flame thickness, it is denoted as the finite thickness expression (FTE).

To further compare these expressions for extrapolation, we expand the extrapolation formula of FTE in terms of the perturbation $\epsilon=1 / r_{f}$ for $\delta^{0} \ll r_{f}$ to the third term as,

$$
\frac{s_{b}}{s_{b}^{0}}=1-2 l_{b} \epsilon-2\left(l_{b}-\delta^{0}\right)^{2} \epsilon^{2}+O\left(\epsilon^{3}\right)
$$

Similarly, we expand Eqs. (2) and (3) as

$$
\begin{aligned}
& \frac{s_{b}}{S_{b}^{0}}=1-2 l_{b} \epsilon+4 l_{b}{ }^{2} \epsilon^{2}+O\left(\epsilon^{3}\right) \\
& \frac{s_{b}}{S_{b}^{0}}=1-2 l_{b} \epsilon-2 l_{b}{ }^{2} \epsilon^{2}+O\left(\epsilon^{3}\right)
\end{aligned}
$$

respectively. It is then seen that all these expressions have the same first and second terms in the expansions, with the difference showing only in the $O\left(\epsilon^{2}\right)$ term. Consequently, in the next section, we will validate the use of Eq. (5) as an extrapolation formula and compare its performance with other methods based on Eqs. (6-8).

\section{Numerical Validations}


In order to test the new extrapolation expression with finite flame thickness, we have conducted numerical simulations with accurate flow description and detailed chemistry. Simulation of the onedimensional expanding spherical flame was performed using the A-SURF code, which has been successfully used in a series of studies on spherical flame initiation and propagation $[4,9,15,16]$. In this code, the compressible conservation equations of one-dimensional, multicomponent, reactive flow in the spherical coordinate are solved using the finite volume method. The CHEMKIN and TRANSPORT packages [17] are incorporated in the A-SURF code to evaluate the chemical reaction rates and thermodynamic data. Also, the one-dimensional adiabatic laminar flame speeds of different fuels in the planar coordinate were calculated using the PREMIX code [18]. Under all conditions, the expanding spherical flames are simulated up to $r_{f}>30 \mathrm{~cm}$ and the simulated flame speeds at the largest flames radius are consistently within $5 \%$ error of the planar flame simulation using PREMIX. The simulated data of $r_{f}$ from $1 \mathrm{~cm}$ to $3 \mathrm{~cm}$ is used as the data for extrapolation, which is typically the range of quasisteady propagation with minimal influence of ignition, chamber confinement and instability $[4,9]$. Thus, by comparing these unstretched values with the extrapolation results, the inaccuracies from different models can be assessed. The hydrogen/air system is selected for the analysis as representative of light fuel, with the detailed chemistry given by the kinetic model of Li et al.[19]; while the $n$-heptane/air mixture is chosen as a typical large hydrocarbon fuel with the kinetic model given in Ref. [20]. In all the simulations, radiation was not considered while multicomponent formulation for the transport properties including Soret diffusion was adopted. 

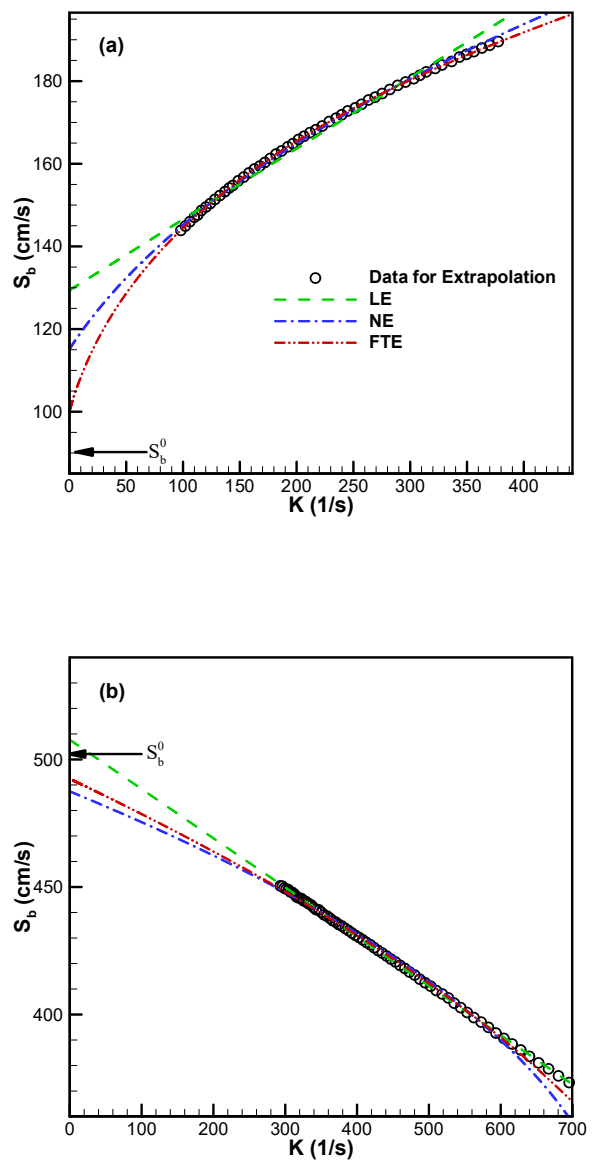

Figure 1: Extrapolation of laminar flame speed at (a) lean condition $(\phi=0.4)$ and $(b)$ rich condition $(\phi=5.0)$ for hydrogen/air mixtures

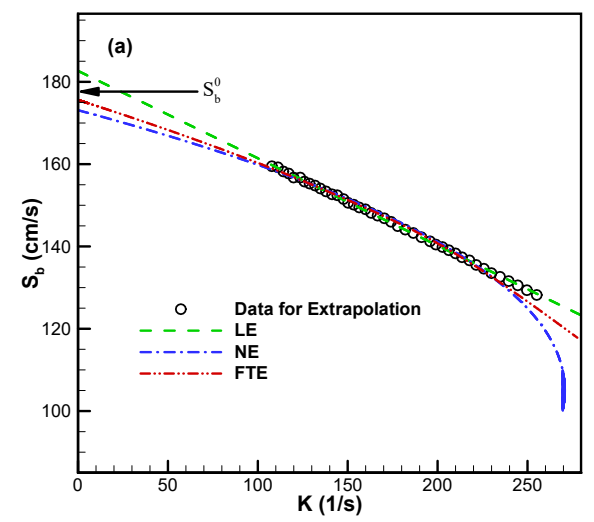




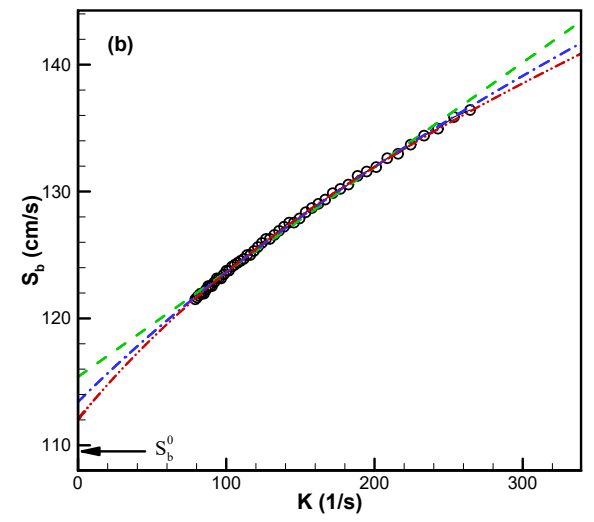

Figure 2: Extrapolation of laminar flame speed at (a) lean condition $(\phi=0.8)$ and (b) rich condition $(\phi=1.7)$ for $n$ heptane/air mixtures

The simulation and extrapolation results for lean $(\phi=0.4)$ and rich conditions $(\phi=5.0)$ are plotted in Fig.1. As expected, for lean (rich) hydrogen/air mixtures the stretched flame speed starts from high (low) values at small radii and hence large stretch, respectively. As the flame grows and the stretch rate decreases, the flame speed calculated for lean (rich) mixtures with negative (positive) Markstein lengths decreases (increases), respectively, approaching the solution from the planar simulation. For the lean condition in Fig.1(a), it is seen that the extrapolation errors for the linear and nonlinear expressions are nearly $42 \%$ and $27 \%$ respectively while extrapolations for the rich condition show smaller discrepancies, see Fig.1(b), with the linear extrapolation having similar accuracy compared with the nonlinear extrapolation, to within 5\% error. It is then noted that the FTE consistently yields the most accurate prediction of the unstretched flame speed, for both the lean and rich mixtures, with extrapolation errors not exceeding $10 \%$. Opposite to hydrogen, the diffusivity of heavy hydrocarbon fuels is much smaller than nitrogen and oxygen. Consequently, the resulting Markstein length has opposite dependence on the equivalence ratio. Figure 2 plots the extrapolation results of $n$-heptane/air at 1 atm and lean and rich conditions. Since large hydrocarbons decompose quickly in flames, its sensitivity to preferential diffusion is controlled by the diffusion of the fuel fragment, rather than the fuel. Consequently we see 
less nonlinear response to flame stretch than the hydrogen/air flames, although still around $10 \%$ error for LE is caused by the extrapolation process. Under such conditions, although the FTE prediction is very close to that by NE, we can still see the improvement of extrapolation accuracy by FTE. With the validated performance of FTE applying to lean (rich) hydrogen/air and $n$-heptane/air mixtures, we shall demonstrate that it yields consistently closer predictions than LE and NE.

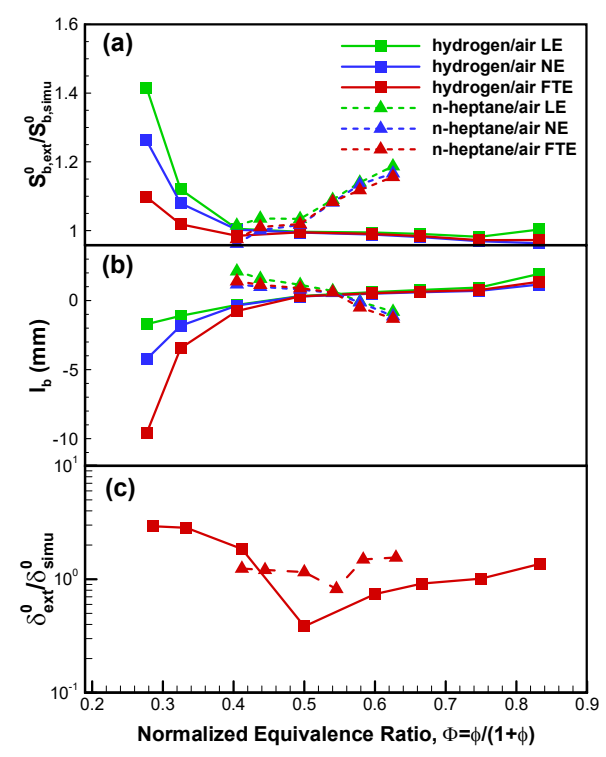

Figure 3: Extrapolation results of (a) laminar flame speed, (b) Markstein length and (c) flame thickness for hydrogen/air and $n$-heptane/air mixtures

Figure 3 shows the extrapolated flame speed $S_{b}^{0}$, Markstein length $l_{b}$, and flame thickness $\delta^{0}$ of the hydrogen and heptane flames respectively; note that we have used the normalized equivalence ratio instead of the equivalence ratio in representing the influence of the fuel/air concentration, recognizing that the former is a more symmetrical and balanced indication of the extent of departure of the mixture concentration from stoichiometry. Figure 3(a) then shows that FTE has the lowest relative difference in the extrapolated flame speed compared with the detailed simulation under all conditions. For the extrapolated $l_{b}$ given in Fig. 3(b), since there is no absolute measure of this quantity as compared to $S_{b}^{0}$, the values shown are dependent on the expressions used in the extrapolation, which in turn depends on 
the rigor of the derivations of these expressions. Consequently more significance should be attached to the values extrapolated by using the FTE. It is then of interest to see that while all three expressions show similar values of $l_{b}$ for the heptane mixtures, the FTE consistently yields larger $l_{b}$, especially for very lean hydrogen mixtures, demonstrating its heightened sensitivity to stretch. Finally, to get a sense of the magnitude of the extrapolated $\delta^{0}$ from FTE, and recognizing that this quantity is not present in the LE and NE, we compare in Fig. 3(c) the extrapolated $\delta^{0}$ with the flame thickness determined from the simulated result, frequently defined as $\delta_{\text {simu }}^{0}=\left(T_{a d}-T_{u}\right) / \max (d T / d x)$. Following the theoretical results in Ref. [13], it should be noted that $\delta^{0}$ extrapolated using FTE must be further corrected by the thermal expansion ratio considering the density variation. It is then seen that $\delta^{0}$ is of the same magnitude as the simulated ones, with the hydrogen case again exhibiting stronger sensitivity to the definitions. The final point to note is that the extrapolated flame thickness can be determined from the measured stretched flame speed data by using FTE, in the course of extrapolating the laminar flame speed, without the need to separately measure/simulate the entire thermal structure of the stretched flame and then perform some independent additional extrapolation. Thus the present approach not only represents significant saving in effort, it is also physically more consistent.

Next, based on these simulation results, we shall compare FTE with other methods according to the expansions in Eqs. (6-8) in order to explain the different performances of LE and NE when $l_{b}$ is positive (negative), and clarify why previous methods fail to predict unstretched flame speed at very rich and lean conditions. Specifically, previous studies $[3,6]$ have shown that when $L e<1\left(l_{b}<0\right)$, the nonlinear expression (3) is more accurate. We notice that for the nonlinear expression (8) the coefficient at the $O\left(\epsilon^{2}\right)$ term is $-2\left|l_{b}\right|^{2}$, which has a large negative contribution, while the linear expression (7) has a positive coefficient, $4\left|l_{b}\right|^{2}$. However for the FTE, when $l_{b}<0$, the coefficient of the $O\left(\epsilon^{2}\right)$ term in (6), $-2\left(l_{b}-\delta^{0}\right)^{2}=-2\left(\left|l_{b}\right|+\delta^{0}\right)^{2}$, has a larger negative contribution compared with the coefficient 
$-2\left|l_{b}\right|^{2}$ in NE. However, the linear expression predicts this term in the wrong direction. So the flame thickening effect makes the nonlinearity of the stretch extrapolation even stronger. This result is consistent with the extrapolation result in Figs. 1(a) and 2(b), which shows that the linear expression is the worst, the nonlinear expression is better and the finite thickness expression yields the most accurate prediction. Also, both linear and nonlinear expressions over-predict the flame speed at such condition as they both have larger second order term than FTE.

However, when $L e>1\left(l_{b}>0\right)$, as mentioned earlier, it was found that the LE over-predicts the laminar flame speed, while the NE under-predicts it [6]. This can also be explained using the $O\left(\epsilon^{2}\right)$ term in Eqs. (6-8). It is noted that, when $l_{b}>0$, the flame thickness $\delta^{0}$ cancels out the positive $l_{b}$ in $-2\left(l_{b}-\right.$ $\left.\delta^{0}\right)^{2}$, the $O\left(\epsilon^{2}\right)$ term of Eq. (6), which makes the coefficient of $O\left(\epsilon^{2}\right)$ term close to zero. So the positive coefficient, $4 l_{b}^{2}$, in the linear expression over-predicts the flame speed, and the negative coefficient, $-2 l_{b}^{2}$, in the nonlinear expression under-predicts it. Consistent with previous studies [4,9], it was found the linear expression without the $O\left(\epsilon^{2}\right)$ term, namely the linear expression based on curvature,

$$
\frac{S_{b}}{S_{b}^{0}}=1-\frac{2 l_{b}}{r_{f}}
$$

could yield an apparently more accurate prediction under such condition. This comparison proves that the finite flame thickness expression is consistent with all other extrapolation expressions for both $L e>1$ and $L e<1$ conditions, and explains the different performances of these expressions in such conditions.

\section{Effects of Pressure}


All the flame responses are known to be strongly affected by pressure [1]. To further demonstrate the performance of different extrapolations, Fig. 4 shows the results of lean hydrogen/air mixtures at pressures lower $(P=0.5 \mathrm{~atm})$ and higher $(P=2.0 \mathrm{~atm})$ than the atmospheric pressure. Comparing between Figs. 4(a) and 4(b), it is seen that the differences among extracted flame speeds decrease with increasing pressure. Furthermore, Fig. 4(a) shows that at sub-atmospheric pressures, both LE and NE substantially over-predict the unstretched flame speed while FTE could still accurately extract it with less than $10 \%$ error. Under such conditions, the flame is thicker than that of atmospheric condition, so the effect of finite flame thickness becomes even more significant than the atmospheric condition, which again substantiates the accuracy and usefulness of using FTE for flame speed extrapolation.
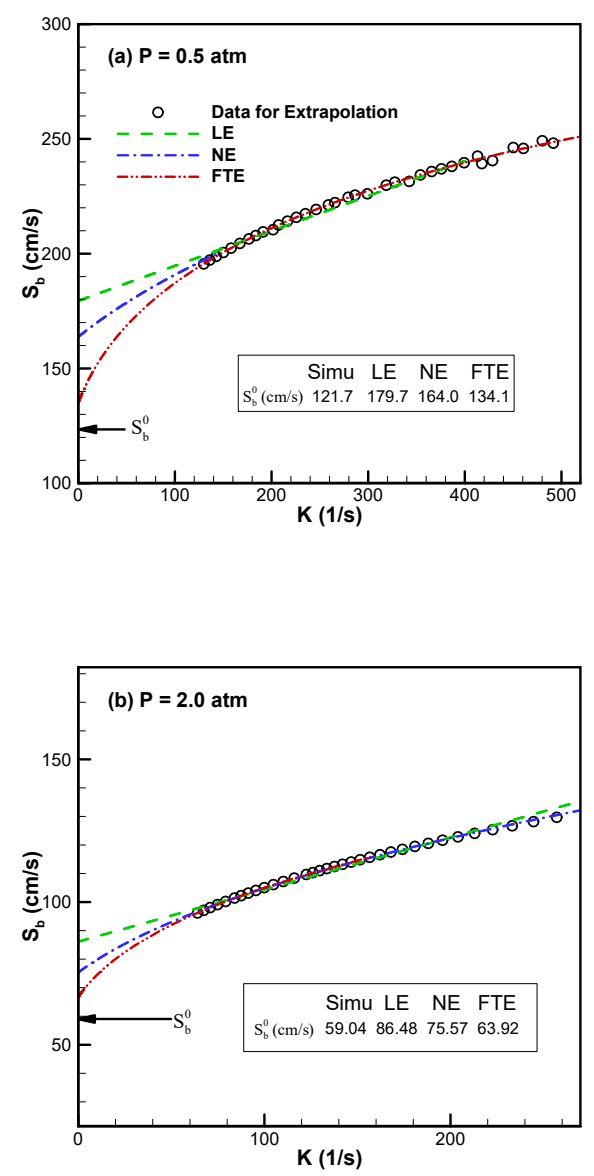

Figure 4: Extrapolation of laminar flame speed at (a) $P=0.5 \mathrm{~atm}$ and (b) $P=2.0 \mathrm{~atm}$ for lean $(\phi=0.4)$ hydrogen/air mixtures 


\section{Effects of Measurement Uncertainty}

To apply the extrapolation expressions to the experimental data, which inevitably contains measurement uncertainty, we shall also test whether an extrapolation expression is robust when considering the uncertainty in the experimental data. Since the simulated data is normally a smooth curve $r_{f, s i m u}(t)$ with no measurement errors as in experiments, we could artificially add some noise to the raw data of flame radius time evolution as follows:

$$
r_{f}=r_{f, \operatorname{simu}}(1+p \cdot G(0,1))
$$

where $G(0,1)$ is a normal distribution with zero mean and unity standard deviation and $p$ is the percentage of noise in the data ranging from $0 \%$ to $8 \%$ in our simulations. After adding random noise into the simulation data, different extrapolation methods could be tested against measurement uncertainty and the results are plotted in Fig.5 for hydrogen/air mixtures at lean $(\phi=0.4)$ conditions. Because the procedure of deriving local flame velocity using discrete differentiation would further magnify the errors in flame radii [21-23], we shall use the integral forms of the extrapolation methods as shown in Ref. [22] or use the procedure presented in Ref. [23], which numerically generate raw data $r_{f}(t)$ from the extrapolation expressions, to achieve better prediction results. From Fig. 5, we can see the growth of the extrapolation uncertainty as the noise in the raw data increases. It is seen that LE is almost not sensitive to measurement noise, but it has the largest extrapolation error for most conditions. NE has the largest growth of the extrapolation error with increasing random noise. FTE not only has the lowest extrapolation error compared with other expressions for small amounts of noise, it also has slower growth rate with increasing random noise in the data compared with NE. This further demonstrates the usefulness of FTE in terms of both accuracy and robustness under measurement noise. 




Figure 5: Extrapolation error of laminar flame speed of lean $(\phi=0.4)$ hydrogen/air mixtures changing with noise percentage

\section{Conclusions}

In this study, we have investigated the assumptions underlying theories describing stretched laminar flame propagation, and recognized that in addition to the laminar flame speed, a finite flame thickness is also needed to give a comprehensive description of the diffusive and reactive nature of laminar flames. Based on these findings, a finite flame thickness expression was adopted and validated at conditions where all previous methods fail to accurately extrapolate the laminar flame speed. Specifically, it is found that the improvement of the extrapolation accuracy using the finite thickness expression is substantial when the Lewis number is very large or very small. Compared with the numerical simulations of rich/lean hydrogen/air and $n$-heptane/air mixtures, the finite thickness flame extrapolation consistently yields more accurate values for the laminar flame speed at various different conditions. Furthermore, the finite thickness expression also explains why the nonlinear extrapolation is more accurate when $L e<1$, as well as why the nonlinear method under-predicts the laminar flame speed, the linear method over-predicts it for $L e>1$.

Another useful attribute of using the finite thickness expression in the extrapolation of the laminar flame speed is that it also yields the collateral flame property of the laminar flame thickness, based on the 
same set of the experimental data on the stretched flame speed. We emphasize again that the laminar flame thickness not only is a functionally independent response of the flame and as such should be independently determined, its information should be embedded in the data on stretched flame speeds as such can be collaterally extracted. Finally, we have also demonstrated that the finite thickness expression is robust in its extrapolation when using raw data with considerable measurement uncertainties, being both accurate and also resistant to random noises inevitably contained in experimental measurements.

\section{Acknowledgments}

This work was supported by the National Science Foundation (CBET,Grant No. 1510142). WL was in addition supported by a Maeder Graduate Fellowship at Princeton University.

\section{References}

[1] C.K. Law, Combustion Physics, Cambridge University Press, New York, 2006.

[2] F.N. Egolfopoulos, N. Hansen, Y. Ju, K. Kohse-Hoinghaus, C.K. Law, F. Qi, Prog. Energy Combust. Sci. 43 (2014) 36-67.

[3] A.P. Kelley, C.K. Law, Combust. Flame 156 (2009) 1844-1851.

[4] Z. Chen, Combust. Flame 158 (2011) 291-300.

[5] A.P. Kelley, J.K. Bechtold, C.K. Law, J. Fluid Mech. 691 (2012) 26-51.

[6] F. Wu, W. Liang, Z. Chen, Y. Ju, C.K. Law, Proc. Combust. Inst. 35 (2015) 663-670.

[7] E. Varea, J. Beeckmann, H. Pitsch, Z. Chen, B. Renou, Proc. Combust. Inst. 35(2015) 711-719.

[8] J. Jayachandran, R. Zhao, F.N. Egolfopoulos, Combust. Flame (2014) 2305-2316.

[9] Z. Chen, Combust. Flame 162 (2015) 2442-2453.

[10] C.K. Wu, C.K. Law, Proc. Combust. Inst. 20 (1984) 1941-1949.

[11] M. Matalon, B.J. Matkowsky, J. Fluid Mech. 124 (1982) 239-259. 
[12] P.D. Ronney, G.I. Sivashinsky, SIAM J. Appl. Math.49 (1989) 1029-1046.

[13] M.L. Frankel, G.I.Sivashinsky, Combust. Sci. Tech., 31(1983) 131-138.

[14] Z. Chen, Y. Ju, Combust. Theory Modell., 11 (2007) 427-453.

[15] W. Liang, Z. Chen, F. Yang, H. Zhang, Proc. Combust. Inst., 34 (2013) 695-702.

[16] H. Yu, W. Han, J. Santner, X.L. Gou, C.H. Sohn, Y.G. Ju, Z. Chen, Combust. Flame, 161 (2014) $2815-2824$.

[17] R.J. Kee, F.M. Rupley, J.A. Miller, Sandia National Laboratories Report, (1989).

[18] R.J. Kee, J.F. Grcar, M.D.Smooke, J.A. Miller, E. Meeks, Sandia National Laboratories Report, (1985).

[19] J. Li, Z. Zhao, A. Kazakov, F.L. Dryer, Int. J. Chem. Kinet., 36 (2004) 566-575.

[20] C.S. Yoo, T. Lu, J.H. Chen, C.K. Law, Combust. Flame, 158 (2011) 1727-1741.

[21] F. Halter, T. Tahtouh, C. Mounaïm-Rousselle, Combust. Flame, 157 (2010) 1825-1832.

[22] A.N. Lipatnikov, S.S. Shy, W.Y. Li, Combust. Flame, 162 (2015) 2840-2854.

[23] S. Coronel, N. Bitter, V. Thomas, R. Mevel, J. E. Shepherd, Non-linear extrapolation of laminar flame properties from spherically expanding flames, Paper 087LF-0020, Western States Section of the Combustion Institute, March 24-25 2014. 


\section{Figure Captions:}

Figure 1: Extrapolation of laminar flame speed at (a) lean condition $(\phi=0.4)$ and (b) rich condition $(\phi=5.0)$ for hydrogen/air mixtures

Figure 2: Extrapolation of laminar flame speed at (a) lean condition $(\phi=0.8)$ and (b) rich condition $(\phi=1.7)$ for n-heptane/air mixtures

Figure 3: Extrapolation results of (a) laminar flame speed, (b) Markstein length and (c) flame thickness for hydrogen/air and n-heptane/air mixtures

Figure 4: Extrapolation of laminar flame speed at (a) $\mathrm{P}=0.5 \mathrm{~atm}$ and (b) $\mathrm{P}=2.0 \mathrm{~atm}$ for lean $(\phi=0.4)$ hydrogen/air mixtures

Figure 5: Extrapolation error of laminar flame speed of lean $(\phi=0.4)$ hydrogen/air mixtures changing with noise percentage 